Atomic data and the spectrum of the solar photosphere

This article has been downloaded from IOPscience. Please scroll down to see the full text article.

1993 Phys. Scr. 1993133

(http://iopscience.iop.org/1402-4896/1993/T47/021)

View the table of contents for this issue, or go to the journal homepage for more

Download details:

IP Address: 139.165.38.134

The article was downloaded on 14/10/2012 at 20:06

Please note that terms and conditions apply. 


\title{
Atomic Data and the Spectrum of the Solar Photosphere
}

\author{
N. Grevesse and A. Noels \\ Institut d'Astrophysique, Université de Liège, 5, avenue de Cointe, B-4000 Liege, Belgium
}

Received October 14, 1992; accepted in revised form February 12, 1993

\begin{abstract}
Even though the solar photospheric abundances are now on the whole in very good agreement with the meteoritic values, some problems remain which will only be solved with a higher level of accuracy in the atomic data, especially the transition probabilities for faint lines. We justify this request for many more very accurate $g f$-values through a few particularly striking points.
\end{abstract}

\section{Introduction}

The study of the photospheric spectrum teaches us important aspects of various problems that are suspected to be encountered in other stars as well. Since the Sun is the brightest star, it is the one for which the observations reach the highest signal-to-noise ratio and resolving power.

The interpretation of these data should lead to the refined knowledge of the physical conditions, physical processes and abundances of the elements in the upper layers of the Sun. As long as these problems are not entirely solved for the Sun, we will not be able to handle similar problems for other stars with success.

It is well known that these problems are interconnected and strongly depend on basic atomic data: wavelengths, ionization energies, partition functions, hyperfine structures, line broadening parameters, Landé $g$-values, cross-sections and transition probabilities. These aspects have already been discussed in the past [1-3]. We shall update these reviews, insisting on a few crucial points like the wavelengths and transition probabilities. Other aspects of atomic data, also needed in solar spectroscopy, are covered in the contributions of L. Andersen, R. L. Kurucz and D. L. Lambert to this volume as well as in [3].

\section{Wavelengths}

\subsection{Line identifications}

Very high quality solar spectra at the center of the solar disk are now available from $300 \mathrm{~nm}$ to $16 \mu \mathrm{m}$ [4-6]. Photospheric UV spectra also exist but they will not be discussed here, as they do not help very much in solving the problems mentioned in the introduction because of too numerous blends below $3000 \AA$.

Kurucz $[7,8]$ has drawn attention to the fact that a very large number of solar lines are still unidentified or unclassified in the visible spectrum, even when using his new data which include millions of new lines [9]. On the other hand, using his numerous new results for faint lines of the iron group, he has been able to solve the problem of the "missing UV opacity" [10].

In the infrared, the situation is even worse than in the visible. Geller [11] has recently identified a large number of lines in the ATMOS solar infrared spectra obtained from space and covering the region from 2.5 to $16 \mu \mathrm{m}$ (646 to $4798 \mathrm{~cm}^{-1}$ ). Molecular lines are all attributed without any doubt but among the atomic lines, quite a large number of lines remain unidentified due to the lack of laboratory data for atomic species in the IR.

The solar spectrum has eventually been used as a laboratory source to provide important additional information concerning highly excited levels in C I, Mg I, Si I, Ca I, Fe I $[11,12]$. Most of these levels have never been observed from laboratory sources.

\subsection{Precision}

Solar granulation is the evidence of motions in the upper layers of the Sun, hot matter moving upwards while cool matter moves downward. Observations indeed show that lines, after correction for the Sun-Earth motion and for the gravitational redshift, are blue shifted and asymmetric [1318].

These apparent Doppler shifts are key data and severe constraints for modelling the detailed dynamical structure of the photosphere. As these Doppler velocities vary from a few tens of $\mathrm{m} / \mathrm{s}$ to a few hundreds of $\mathrm{m} / \mathrm{s}$, the accuracy of the laboratory wavelengths of the unblended solar lines used for these diagnostics must accordingly be of the order of $\delta \lambda /$ $\lambda \sim 10^{-7}$, i.e. $30 \mathrm{~m} / \mathrm{s}$ (see also [19]).

\section{Transition probabilities}

It is well known that the accuracy of the transition probabilities play a key role not only for solar abundance determinations but also to test the empirical model of the photosphere (see e.g. [2]), to derive accurate values of the microturbulence [20] and to test hypothesis of local thermodynamic equilibrium, LTE (see e.g. $[2,19]$ ).

We shall hereafter discuss the impact of the accuracy of the transition probabilities on the solar abundances and the needs for highly accurate $g f$-values for many more faint lines of neutral and once ionized atomic species present in the solar photospheric spectrum.

\subsection{Need for accurate solar abundances}

Among the many reasons why we continue to refine the solar photospheric abundances (see e.g. [21-24]), are some very important points.

It has been a longstanding problem to know whether there is a difference or not between the solar photospheric abundances and the meteoritic ones. Large discrepancies previously found have progressively disappeared as the accuracy of the transitions probabilities has been increasing. Anders and Grevesse [25] have shown that on the 
whole the agreement is remarkably good between photospheric and meteoritic abundances. However, the uncertainties are much larger for the solar results than for the meteorites and it is still impossible to rule out slight differences hidden in the solar photospheric error bars. These error bars (see Section 3.4) essentially come from the uncertainties in the transition probabilities.

Photospheric abundances are essential data in the opacity computations used in models of the external layers of the Sun as well as in evolutionary models of the Sun's interior, if it can be proven that they reflect the chemical composition of the solar nebula. For other stars, the distribution of elements heavier than helium in the metallicity is often assumed to be solarlike. After the pioneering work of computing an Astrophysical Opacity Library by the Los Alamos group [26], gigantic efforts have recently been made to considerably improve the atomic data and the equation of state for stellar interiors as well as for envelopes. Two groups have directed their efforts towards high temperature opacities (OPAL: [27]; Opacity Project: [28, 29]). while Kurucz [9] computed opacities to be used in stellar atmosphere modelling. Such improvements in the opacity computations demand very accurate abundances for all the numerous contributors to these opacities. As an example, the reduction of the iron photospheric abundance to the meteoritic value (see Section 3.2) has a significant influence on the opacities, even at physical conditions typical of the solar central regions [30], and change the calibrated solar model.

It is now well known that there are differences between the photospheric and the coronal abundances due to a fractionation process leading to an overabundance in the corona of the elements which have their first ionization potential lower than about $10 \mathrm{eV}$. Even more subtle behaviours among different elements are now observed and they must be carefully analysed before being used as constraints in any hydrodynamical envelope model.

There might possibly exist diffusion mechanisms which could lead to slight abundance gradients for some elements in the photosphere. Unfortunately, it is still out of reach to observe such gradients, since the dispersion in the photospheric results is still much too large. It would require much higher accuracy in the $g f$-values for many more faint lines.

\subsection{Photospheric abundance results}

Progress made during the last decade or so in our knowledge of the photospheric abundances is essentially due to the availability of accurate $g f$-values for lines of solar interest. As already mentioned in Section 3.1, past discrepancies between meteoritic and photospheric abundances have disappeared as accurate transition probabilities have progressively become available for many more species and many more lines. Atomic spectroscopists have played a major role in allowing to refine the photospheric abundances and they are co-authors of many papers on solar abundances.

Meteoritic abundances are now known with an accuracy often better than $10 \%$ [25]. Photospheric abundances, as reviewed in [25] and in different more recent papers [22-24] still have much larger error bars (see Section 3.4).

The longstanding puzzling problem of the difference between the photospheric and the meteoritic abundance of iron has recently been solved. Let us recall that Blackwell et al. $[31,32]$, using the very accurate Oxford transition probabilities [33] for low excitation $\mathrm{Fe} I$ lines, derived a solar abundance of iron of $A_{\mathrm{Fe}}=7.67 \pm 0.03$ (where $A_{\mathrm{el}}=$ $N_{\mathrm{el}} / N_{\mathrm{H}}$, the abundance by number relative to hydrogen in the usual logarithmic scale where $\log N_{\mathrm{H}}=12.00$ ), larger than the very accurate meteoritic value of $A_{\mathrm{Fe}}=7.51 \pm 0.01$ [25].

New accurate transition probabilities have recently been obtained for lines of higher excitation in $\mathrm{Fe} \mathrm{I}$ as well as for Fe II lines. Such lines are much less sensitive to departure from LTE and to slight uncertainties in the atmospheric model than the low excitation $\mathrm{Fe} I$ lines. Different groups, including the atomic spectroscopists who obtained the new $g f$-values, have redetermined the photospheric abundance of iron using these new data [34-38]. The new results all converge to the meteoritic value with uncertainties of the order of 10 to $15 \%$. Even if the problem seems to be solved, we claim that more analyses should be done using $\mathrm{Fe} I$ as well as $\mathrm{Fe}$ II lines. To obtain significant results, we need many more very accurate $g$ f-values for faint $\mathrm{Fe}$ I and Fe II lines (see Section 3.4).

Rather puzzling are recent results on Sc [39] and on $\mathrm{Ti}$ [40] abundances, obtained using accurate $g f$-values for both neutral and singly ionized species. These solar abundances are slightly larger than the meteoritic values.

Even if the agreement between photospheric and meteoritic abundances on the whole is very good, we still need to use many more faint lines of very good quality with large ranges of excitation energies in order to seek for possible differences between the meteorites and the solar photosphere and to better understand the physical processes in the photosphere.

\section{3. $g f$-values and physical processes}

We summarized in [3] the problem of the empirical departures from LTE observed in iron group neutrals thanks to the use of very accurate $g f$-values (a few \%). Figure 1 shows the abundance results obtained from low excitation lines in Fe I [31], Cr I [41], Ti I [41, 42] and V I [43].

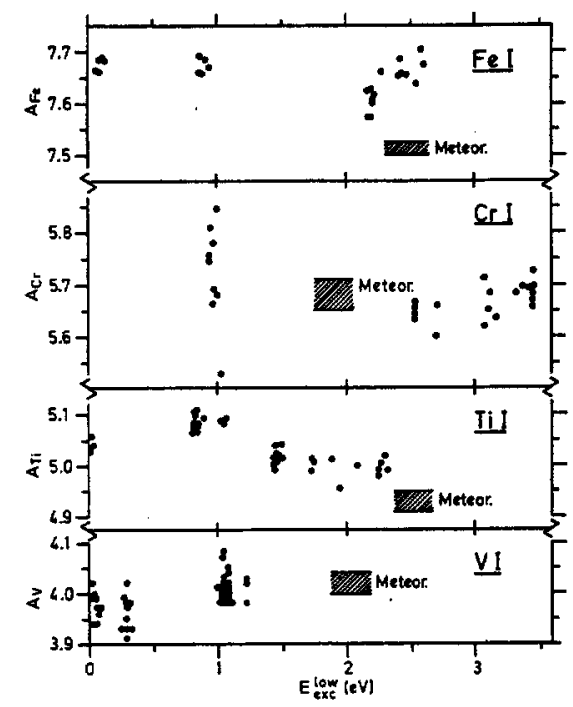

Fig. 1. Solar abundances, as functions of the excitation energy, derived using very accurate $g f$-values for low excitation lines of $\mathrm{Fe} \mathrm{I} \mathrm{[31],} \mathrm{Cr} \mathrm{I} \mathrm{[41],}$ Ti I $[41,42]$ and $V$ I [43]. The variations of the abundance with excitation energy are real and correspond to non-LTE effects (see text) 
For narrow ranges of the excitation energy, the dispersion of the abundance results is quite small but there is clearly a trend showing some dependence of the abundance on the excitation energy of the lower level. This could possibly be explained as slight and subtle departures from LTE. These peculiar behaviours would totally be masked if less accurate $g f$-values had been used (see Section 3.4).

\section{4. gf-values and abundances}

We shall hereafter illustrate with a few examples the influence of the accuracy of the $g f$-values on the dispersion and the mean value of the solar photospheric abundances.

Figure 2 shows the vanadium abundance as a function of the excitation energy as derived by different authors ([4446], presents results: [43]), using the $g f$-values available at the time. Note that there is a strong decrease in the dispersion of the results down to the very small dispersion in [43] obtained when using accurate $g f$-values derived from lifetime and branching fraction measurements.

The iron abundance has followed the same trend with time, as can be seen in Fig. 3 where we have plotted the solar abundances as derived from Fe I lines, using $g f$-values

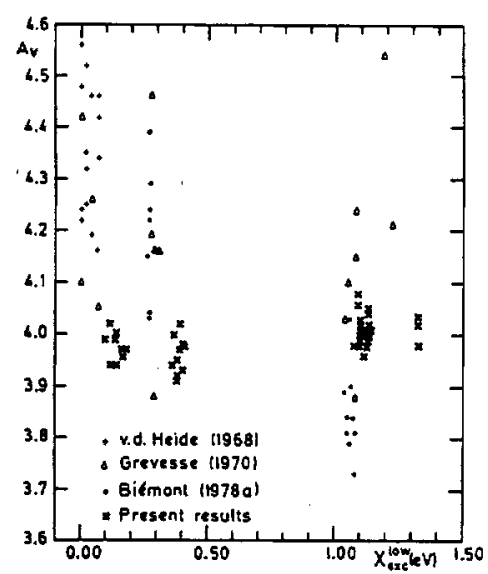

Fig. 2. Abundance of vanadium as a function of the excitation energy derived by different authors [44-46] and present results [43], using different $g f$-values. The very large dispersion noted for works prior to [43] reduces tremendously when using the accurate $g f$-values of [43]. It also shows that results prior to [43] were in complete disagreement with the meteoritic abundance, $A_{\mathrm{V}}=4.02$, essentially because of the use of inaccurate transition probabilities

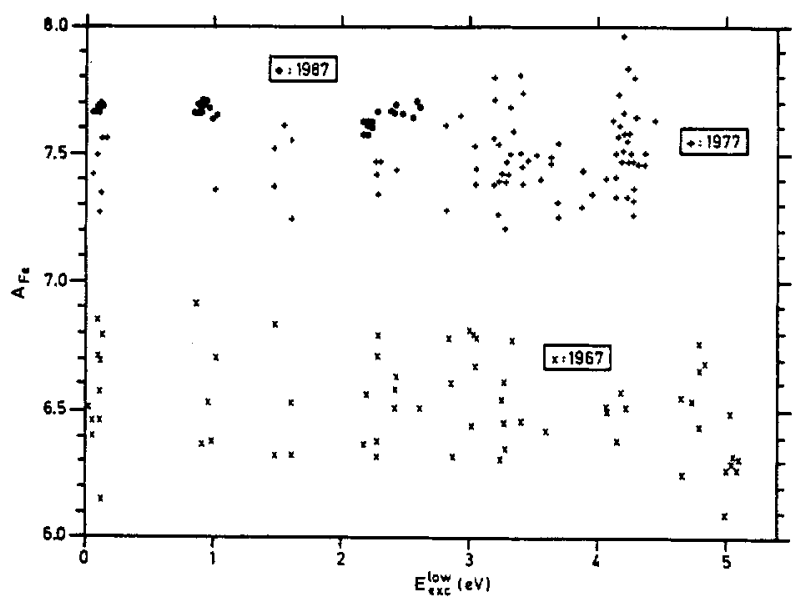

Fig. 3. Evolution of the solar abundance of iron and of the disperson of the results as derived from $\mathrm{Fe} I$ lines. The 1987 results are obtained using the very accurate $g f$-values of Oxford [31]. Earlier results are obtained using solar equivalent widths measured with high accuracy but $g f$-values available in 1967 and 1977 respectively available in 1967,1977 and 1987 . The last results have been obtained with the accurate Oxford $g f$-values [31] (see Section 3.2).

The difference in the mean values and in the dispersion is striking and emphasizes the crucial role played by the accuracy of the transition probabilities.

We must stress that the dispersion observed in solar abundances essentially reflects the level of accuracy of the $g f$-values used.

This iron problem which is now claimed to be solved (see Section 3.2) has in fact opened a new iron problem namely the behaviour of the abundance derived from $\mathrm{Fe} I$ line vs. the excitation energy, $E_{\mathrm{ex}}$, for a very large range of $E_{\mathrm{ex}}$, from 0 to $7.5 \mathrm{eV}$, observed in the solar spectrum. O'Brian $e t$ al. [47] have recently published quite a large number of transition probabilities for $\mathrm{Fe} I$ lines obtained from lifetime and branching fraction measurements. We used their data for about 100 faint Fe I lines in order to avoid the microturbulence and broadening uncertainties. The results are presented in Fig. 4. They are somewhat disappointing in the sense that the dispersion is still quite large. Although the mean value is close to the meteoritic one, the error bar is so large that the agreement could be only apparent. Moreover, because of this large dispersion, nothing definite can be said about the detailed behaviour of the iron abundance in terms of the excitation energy of the lower level. A much smaller dispersion is observed in Fig. 1 (upper panel) obtained with the Oxford $g f$-values [31]. However, it should be recalled that these results lead to a mean value higher than the meteoritic one.

The dispersion observed in Fig. 4 is intimately related to the uncertainty in the $g f$-values, essentially coming from the very difficult technical aspects in the branching fraction measurements for faint lines. Once again, it illustrates that the dispersion in the abundance results is really a function of the uncertainty in the $g f$-values.

When measured branching fractions are missing, some authors turn to Kurucz's semi-empirical $g$ f-values [9], sometimes normalizing them to measured lifetimes. This is not a crucial point as Kurucz's new transition probabilities for the iron group elements are on the whole accurate to within $10-15 \%$ for strong lines which are by far the main contributors to the lifetimes. It is well known that the accuracy of Kurucz's data decreases as the intensity or $g f$-value of the lines decreases (see e.g. $[47,48]$ ). When comparison

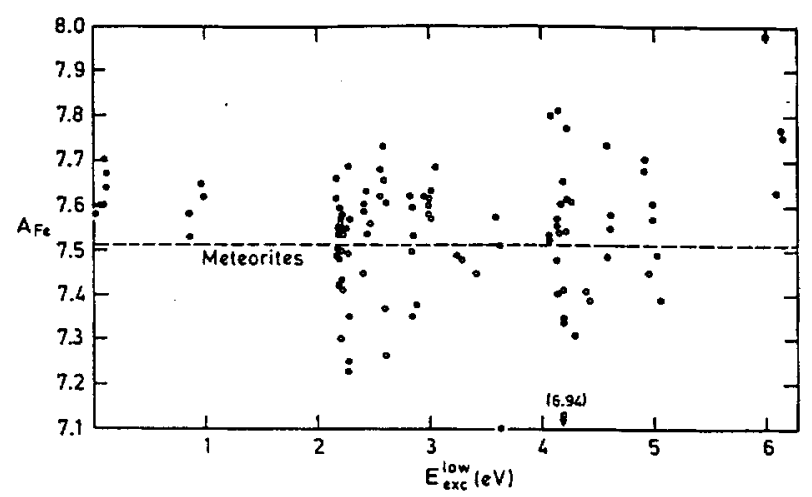

Fig. 4. Solar abundance of iron as derived from a large number of $\mathrm{Fe} I$ lines as a function of the excitation energy up to rather high excitations. The transition probabilities of these essentially faint lines are from [47] (see text) 
can be made, the dispersion obtained with Kurucz's data is much larger than when using measured values. This is illustrated in Fig. 5 which shows the iron abundance derived from Fe II lines with Kurucz's data normalized to the best lifetime values in the upper panel [36] and measured data in the lower one [38]. The mean iron abundance obtained using $g f$-values derived from accurate lifetimes and branching fractions [38] is $A_{\mathrm{Fe}}=7.48 \pm 0.09$ (s.d.) [Fig. 5(A)] whereas using Kurucz's branching fractions [Fig. 5(B)] leads to $A_{\mathrm{Fe}}=7.66 \pm 0.18$ (s.d.). Not only the dispersion of the results is much larger but the mean iron abundance is also much higher. It was only when deleting some lines with high iron abundances that Biémont et al. [36], using Kurucz's $g f$-values [9], obtained a solar abundance in agreement with the meteoritic value.

This is even more striking in Fig. 6 where the Ti abundance derived from Ti II lines is shown using Kurucz's data, normalized to the lifetime of [40] (upper panel B), and $g f$ values obtained from accurate lifetime and branching fraction measurements (lower panel A) [40]. The solar abundance of $\mathrm{Ti}$ is $A_{\mathrm{Ti}}=5.04 \pm 0.04$ [Fig. 6(A)]; it becomes $A_{\mathrm{Ti}}=4.97 \pm 0.30$ with Kurucz's data.

These results are not shown to criticize Kurucz's $g f$-values [9]. Kurucz's data have been computed for opacity purposes and not for a line by line analysis. The same type of remark could be made concerning the Opacity Project $g f$ -

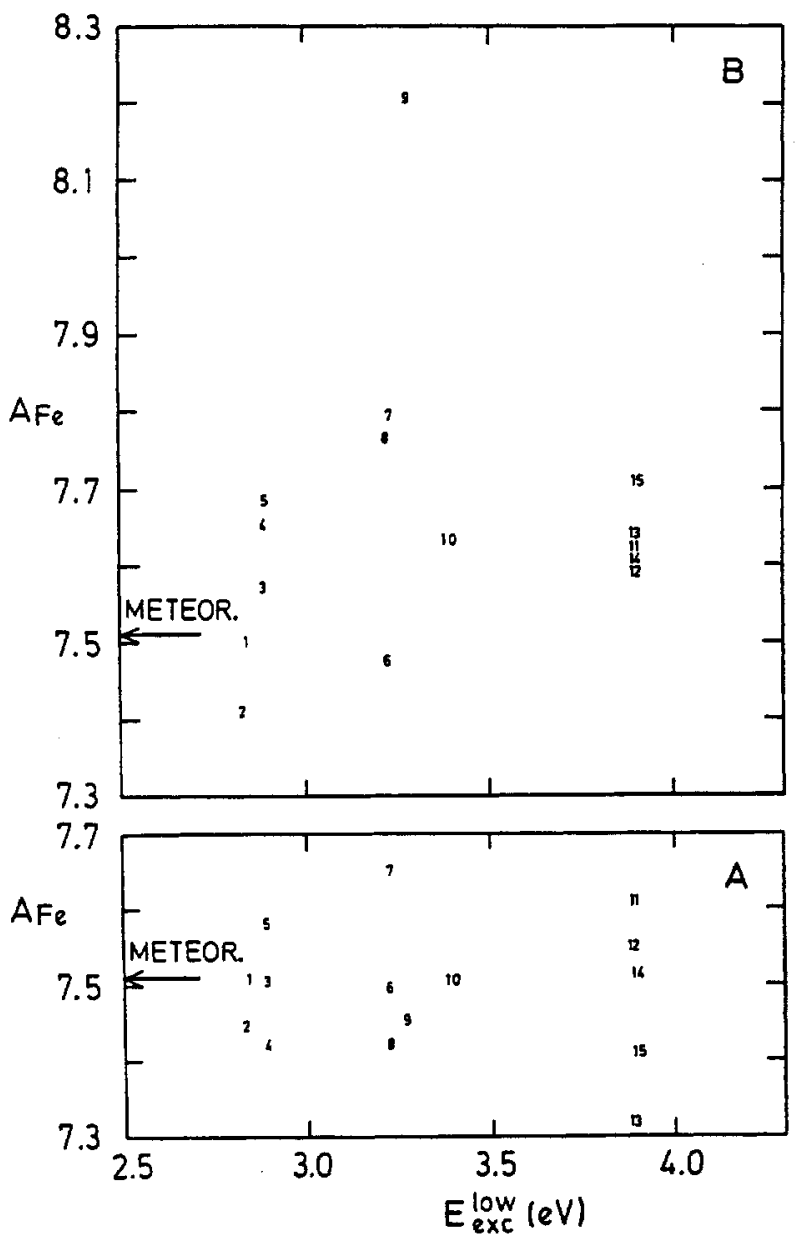

Fig. 5. Solar abundance of iron as derived from Fe II lines as a function of the excitation energy. Panel $A$ shows the results obtained using measured $g f$-values from [38]. Panel B displays the same lines but using Kurucz's semi-empirical $g f$-values [9]. The lines are numbered as they appear in Table 4 of [38] (see text)

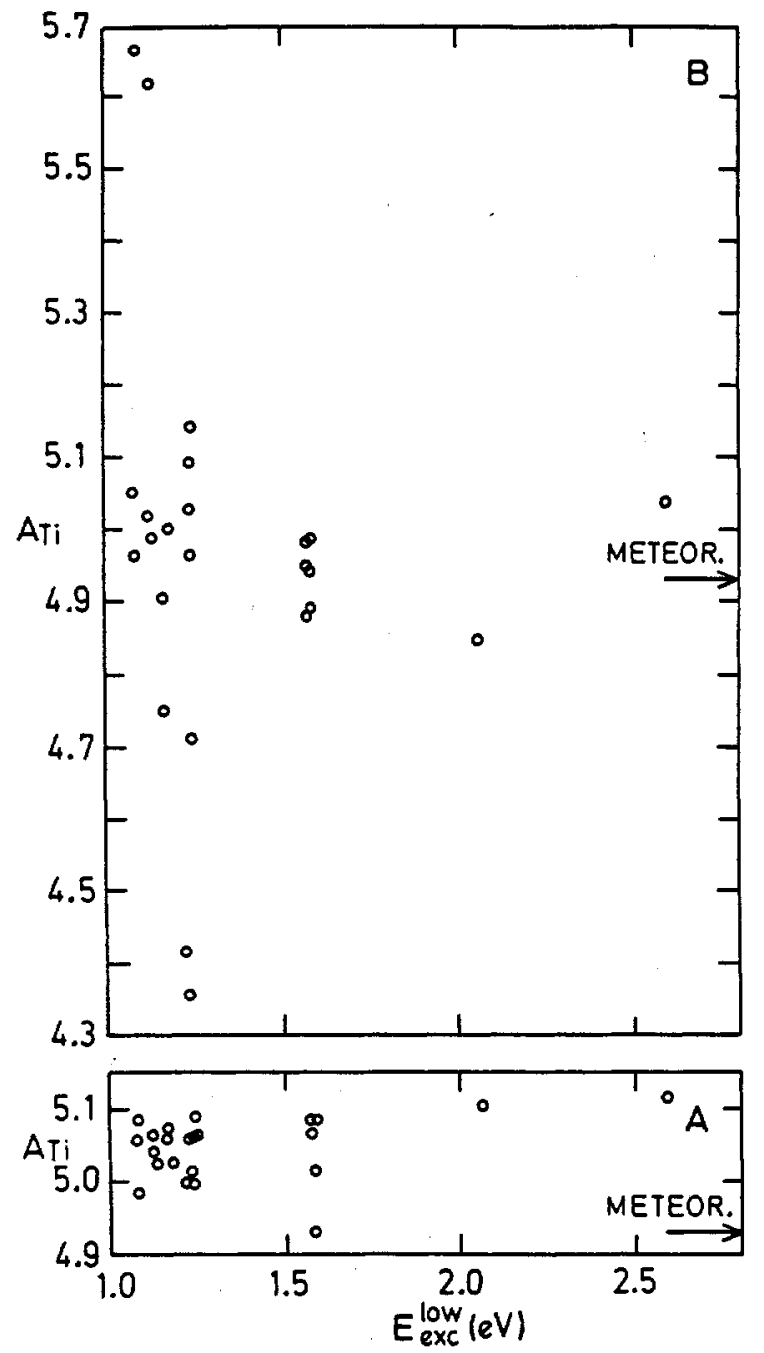

Fig. 6. Solar abundance of titanium as derived from Ti II lines as a function of the excitation energy. Panel A shows the results obtained using measured $g f$-values from [40]. Panel B displays the same lines but using Kurucz's semi-empirical $g$-values [9] (see text)

values $[28,29]$. Using these data for abundance determinations from faint lines might be seriously misleading.

Sometimes, only one line is a available to derive the solar abundance of an important element. As an example, thorium shows only one Th II line, at $4019.13 \AA$. Now, the abundance of this element, as derived from this single line that is present in solar and stellar spectra, is a chronometer for determining the age of the Galaxy [49]. It has been shown that this line is blended with a Co I line whose $g f-$ value has been measured with accuracy [50]. The contribution of this Co I line to the Th II feature is not at all negligible and affects the age determination of the Galaxy. Now, even taking this known blend into account, the solar abundance of thorium is about $40 \%$ larger than the meteoritic abundance. As thorium is a refractory element, there is no reason why such a difference should exist [25]. One possible explanation is the presence of another blend. A likely candidate is the intercombination line of $\operatorname{VI}\left(a^{2} D_{5 / 2}\right.$ $\left.-w^{4} P_{5 / 2}\right)$ with $\log g f=-1.999$ as computed by Kurucz [9]. With such a strength, the solar contribution of the VI line to the Th II feature is too small to bring the thorium abundance down to the meteoritic value. However, it has been shown [43] that Kurucz's results for intercombination lines in VI might be much too small. Solar and stellar spec- 
troscopists urgently need accurate measurements of this single VI line.

\section{Conclusions}

It is now generally accepted that the solar photospheric abundances agree with the meteoritic values thanks to the increase in the number and accuracy of measured transition probabilities for many species. However, this does not mean that the atomic data available at present allow us to fully understand the solar photospheric spectrum.

- Quite a large number of lines are still unidentified or unclassified.

- A higher accuracy in the laboratory wavelength is needed.

- Some problems remain in the photospheric abundances of some species which are still different from the meteoritic values.

- As the accuracy of the $g f$-values increases further, more and more sophisticated informations will be extracted from the solar spectrum.

- Even when the solar photospheric abundances agree with the meteoritic values, the dispersion of the solar results is generally rather large; this could hide some extremely valuable informations on the physics of the photosphere.

- The dispersion observed in solar photospheric abundances essentially reflects the level of accuracy of the $g f$ values used. Astronomers urgently need higher accuracy measurements of branching fractions for faint lines.

Many more high accuracy $g f$-values for a larger number of lines are certainly needed for Mg I, Al I, P I, S I, Mn I, $\mathrm{Mn} \mathrm{II,} \mathrm{Fe} \mathrm{I,} \mathrm{Fe} \mathrm{II,} \mathrm{Cu}$ I, $\mathrm{Zn} \mathrm{I}, \mathrm{Ba}$ II and the rare earths.

\section{Acknowledgements}

We would like to thank A. Bizzarri, J. W. Brault, M. Geller, P. Hannaford, M. C. E. Huber, S. Johansson, R. L. Kurucz, J. E. Lawler, M. Lowe, A. J. Sauval and $W$. Whaling for collaborations and helpful discussions. This work was supported by the Belgian Fonds National de la Recherche Scientifique.

\section{References}

1. Biémont, E. and Grevesse, N., Physica Scripta 16, 39 (1977).

2. Grevesse, N., Physica Scripta T8, 49 (1984).

3. Grevesse, N., in: "Third Atomic Data Workshop" (Edited by C. J. Zeippen and M. C. Le Dourneuf) [J. de Phys. IV, Coll. C1, 181 (1991)].

4. Delbouille, L., Roland, G. and Neven, L., "Photometric Atlas of the Solar Spectrum from $\lambda=3000 \AA$ to $10000 \AA$ " (Institut d'Astrophysique, Université de Liège 1973).

5. Delbouille, L., Roland, G., Brault, J. W. and Testerman, L., "Photometric Atlas of the Solar Spectrum from 1850 to $10000 \mathrm{~cm}^{-1 "}$ (Kitt Peak National Observatory, Tucson 1981).

6. Farmer, C. B. and Norton, R. H., "A High-Resolution Atlas of the Infrared Spectrum of the Sun and the Earth Atmosphere from Space. Vol. I: The Sun" (NASA Ref. Publ. 1224) (NASA, Washington D.C. 1989).

7. Kurucz, R. L., in: "Colloquium on Atomic Spectra and Oscillator Strengths for Astrophysics and Fusion Research" (Edited by J. E. Hansen) (Kon. Ned. Akad. Wetensch, Amsterdam 1990), p. 57.

8. Kurucz, R. L., in: "Workshop on Astrophysical Opacities" (Edited by A. E. Lynas-Gray, C. Mendoza and C. J. Zeippen) [Rev. Mex. Astron. Astrofis. 23, 187 (1992)].

9. Kurucz, R. L., in: "Transactions IAU" (Edited by D. McNally) (Kluwer, Dordrecht 1989), Vol. XXB, p. 168.
10. Kurucz, R. L., in: "Workshop on Astrophysical Opacities" (Edited by A. E. Lynas-Gray, C. Mendoza and C. J. Zeipen) [Rev. Mex. Astron. Astrofis. 23, 181 (1992)].

11. Geller, M., "A High-Resolution Atlas of the Infrared Spectrum of the Sun and the Earth Atmosphere from Space. Vol. III: Key to Identification of Solar Features" (NASA Ref. Publ. 1224) (NASA, Washington D.C. 1992).

12. Johansson, S., Nave, G., Geller, M., Sauval, N. and Grevesse, N., in: "Infrared Solar Physics" (IAU Symposium 154) (Edited by D. Rabin and J. T. Jefferies) (Kluwer, Dordrecht 1993) in press.

13. Dravins, D., Lindegren, L. and Nordlund, A., Astron. Astrophys. 96, 345 (1981).

14. Dravins, D., Ann. Rev. Astron. Astrophys. 20, 61 (1982).

15. Dravins, D., Larsson, B. and Nordlund, A., Astron. Astrophys. 158, 83 (1986).

16. Nadeau, D., Astrophys. J. 325, 480 (1988).

17. Grevesse, N. and Sauval, A. J., in: "The Infrared Spectral Region of Stars" (Edited by C. Jaschek and Y. Andrillat) (Cambridge University Press, Cambridge 1991), p. 215.

18. Blomme, R., Sauval, A. J. and Grevesse, N., in: "Infrared Solar Physics" (Edited by D. Rabin and J. T. Jefferies) (IAU Symposium 154) (Kluwer, Dordrecht 1993) in press.

19. Anderson, L., this volume (1993).

20. Blackwell, D. E., Ibbetson, P. A., Petford, A. D. and Willis, R. B., Mon. Not. Roy. Astr. Soc. 177, 227 (1976).

21. Grevesse, N., in: "Frontiers of Astronomy and Astrophysics" (Edited by R. Pallavicini) (Italian Astronomical Society, Florence 1984), p. 71.

22. Grevesse, N., in: "Evolution of Stars: the Photospheric Abundance Connection" (Edited by G. Michaud and A. Tutukov) (Kluwer, Dordrecht 1991), p. 63

23. Grevesse, N. and Noels, A., in: "Origin and Evolution of the Elements" (Edited by N. Prantzos, E. Vangioni-Flam and M. Cassé) (Cambridge University Press, Cambridge 1993) in press.

24. Grevesse, N., Noels, A. and Sauval, A. J., "Coronal Streamers, Coronal Loops, and Coronal and Solar Wind Composition" (ESA Publ. SP-348, 1992), p. 305.

25. Anders, E. and Grevesse, N., Geochim. Cosmochim. Acta 53, 197 (1989).

26. Huebner, W. F., Merts, A. L., Magee, N. H. Jr. and Argo, M. F., "Astrophysical Opacity Library" (Los Alamos Sci. Lab. Rep. LA 6760, 1977).

27. Iglesias, C. A. and Rogers, F. J., in: "Workshop on Astrophysical Opacities" (Edited by A. E. Lynas-Gray, C. Mendoza and C. J. Zeippen) [Rev. Mex. Astron. Astrofis 23, 161 (1992)].

28. Seaton, M. J., Zeippen, C. J., Tully, J. A., Pradhan, A. K., Mendoza, C., Hibbert, A. and Berrington, K. A., in: "Workshop on Astrophysical Opacities" (Edited by A. E. Lynas-Gray, C. Mendoza and C. J. Zeippen) [Rev. Mex. Astron. Astrofis 23, 19 (1992)].

29. Mendoza, C., this volume (1993).

30. Iglesias, C. A., Rogers, F. J. and Wilson, B. G., Astrophys. J. 397, 717 (1992).

31. Blackwell, D. E., Booth, A. J. and Petford, A. D., Astron. Astrophys., 132, 236 (1984).

32. Blackwell, D. E., Booth, A. J., Haddock, D. J., Petford, A. D. and Leggett, S. K., Mon. Not. Roy. Astr. Soc. 220, 549 (1986).

33. Blackwell, D. E., Petford, A. D. and Simmons, G. J., Mon. Not. Roy. Astr. Soc. 201, 595 (1982) and references therein.

34. Holweger, H., Heise, C. and Kock, M., Astron. Astrophys. 232, 510 (1990).

35. Pauls, U., Grevesse, N. and Huber, M. C. E., Astron. Astrophys. 231, $536(1990)$

36. Biémont, E., Baudoux, M., Kurucz, R. L., Ansbacher, W. and Pinnington, E. H., Astron. Astrophys. 249, 539 (1991).

37. Holweger, H., Bard, A., Kock, A. and Kock, M., Astron. Astrophys. 249, 545 (1991).

38. Hannaford, P., Lowe, R. M., Grevesse, N. and Noels, A., Astron. Astrophys. 259, 301 (1992).

39. Neuforge, C., in: "Origin and Evolution of the Elements" (Edited by N. Prantzos, E. Vangioni-Flam and M. Cassé) (Cambridge University Press, Cambridge 1993), in press.

40. Bizzarri, A., Huber, M. C. E., Noels, A., Grevesse, N., Bergeson, S. D., Tsekeris, P. and Lawler, J. E., Astron. Astrophys. in press (1993).

41. Blackwell, D. E., Booth, A. J., Menon, S. L. R. and Petford, A. D., Astron. Astrophys. 180, 229 (1987) 
42. Grevesse, N., Blackwell, D. E. and Petford, A. D., Astron. Astrophys. 208, 157 (1989).

43. Whaling, W., Hannaford, P., Lowe, R. M., Biémont, E. and Grevesse, N., Astron. Astrophys. 153, 109 (1985).

44. von der Heide, K., Z. Astrophys. 69, 220 (1968).

45. Grevesse, N., Mém. Acad. Roy. Belgique 39, 1 (1970).

46. Biémont, E., Sol. Phys. 56, 79 (1978).
47. O'Brian, T. R., Wickliffe, M. E., Lawler, J. E., Whaling, W. and Brault, J. W., J. Opt. Soc. Am. B8, 1185 (1991).

48. Biémont, E., Grevesse, N., Faires, L. M., Marsden, G., Lawler, J. E. and Whaling, W., Astron. Astrophys. 209, 391 (1989).

49. Butcher, H. R., Nature 328, 127 (1987).

50. Lawler, J. E., Whaling, W. and Grevesse, N., Nature 346, 635 (1990). 